\title{
Understanding the role of electron and hole trions on current transport in aluminium tris(8-hydroxyquinoline) using organic magnetoresistance
}

\author{
Sijie Zhang, ${ }^{1,2}$ M. Willis, ${ }^{1}$ R. Gotto, ${ }^{2}$ K. A. Roy, ${ }^{2}$ N. J. Rolfe, ${ }^{2,3}$ T. Kreouzis, ${ }^{2}$ \\ and W. P. Gillin 1,2,a) \\ ${ }^{1}$ College of Physical Science and Technology, Sichuan University, Chengdu 610064, People's Republic of \\ China \\ ${ }^{2}$ Materials Research Institute and School of Physics and Astronomy, Queen Mary University of London, Mile \\ End Road, London E1 4NS, United Kingdom \\ ${ }^{3}$ University of Surrey, Guildford, Surrey GU2 5XH, United Kingdom
}

(Received 10 December 2013; accepted 16 January 2014; published online 31 January 2014)

\begin{abstract}
The change in current through an organic light emitting diode (OLED) when it is placed in a magnetic field has been dubbed organic magnetoresistance and provides a means to understand the spin interactions that are occurring in working devices. Whilst there are a wide range of interactions that have been proposed to be the cause of the measured effects, there is still a need to identify their individual roles and in particular how they respond to an applied magnetic field. In this work, we investigate the effect of changing the balance of electron and hole injection in a simple aluminium tris(8-hydroxyqinoline) based OLED and demonstrate that the triplet polaron interaction appears to be much stronger for electrons than for holes in this material. (C) 2014 AIP Publishing LLC. [http://dx.doi.org/10.1063/1.4863684]
\end{abstract}

In 2003, it was observed by Kalinowski et al. ${ }^{1}$ that it was possible to change the optical and electrical performance of an organic light emitting diode (OLED) by subjecting it to a weak magnetic field. The change in electroluminescence and current through the device was further studied by Mermer et $a l .^{2}$ and found to be present in a range of devices made from a variety of organic semiconductors. ${ }^{3-12}$ This effect was subsequently called organic magnetoresistance (OMR or OMAR) and has been widely studied in a range of organic materials and device structures. Although there is still much debate in the literature as to the precise mechanisms responsible, it is now widely believed that many of the effects in OLED structures are excitonic in nature and result from perturbations of the spin interactions present in the device upon the application of the magnetic field., ${ }^{1,6}$ However, there is also evidence to show that unipolar processes, such as bipolaron formation, ${ }^{11}$ can also be perturbed by magnetic fields and hence may also play a role in OMR. ${ }^{12}$ The fact that in OLED structures the application of a weak field (e.g., 5-10 mT) can have a significant perturbation on both device current and efficiency implies that the mechanisms responsible are due to spin interactions within the device that affect both the current and efficiency. At present, the device models used in organic semiconductors do not include spin interactions that can be affected by a magnetic field. This is in part because their role in device performance was not believed to be significant but also because there has not been a reliable means of measuring their function in real devices. The ability to use magnetic fields to perturb these spin interactions and measure their effect on device performance therefore provides a technique to identify and characterise these processes so that they can be properly included in device models.

\footnotetext{
${ }^{\text {a) }}$ Author to whom correspondence should be addressed. Electronic mail: w.gillin@qmul.ac.uk
}

Given that many of the mechanisms behind OMR in OLEDs are believed to be based on the presence or formation of excitons, it is vital to understand how modifications in the injection of electron and holes into real devices affects the OMR as this will give us further clues as to the underlying mechanism. One approach to this would be to change the effective work function of either the anode or cathode of a "standard" OLED" ${ }^{13-17}$ and measure how this affects the OMR. In this work, we have deliberately modified both the electron and hole injection into "standard" organic light emitting diode structures to see how these changes affect the measured OMR. We have been able to significantly change the injection barrier for holes through changes in the oxygen plasma treatment of the indium tin oxide (ITO) anode prior to device growth. Similarly, we modify the electron injection by the removal of a $\mathrm{LiF}$ injection layer at the cathode. We demonstrate that whilst both processes modify the efficiency of the resulting device, as a consequence of changing the injected electron/hole ratio, the effect on the OMR is very different with the reduction in electron injection affecting the triplet polaron interaction (TPI) component more significantly than the reduction in hole injection. This suggests that in aluminium tris(8-hydroxyquinoline) $\left(\mathrm{Alq}_{3}\right)$, the triplet polaron interaction may be dominated by electron polarons rather than hole polarons.

The OLEDs consist of an ITO coated glass substrate with a sheet resistivity of $(\sim 13 \Omega / \square), 50 \mathrm{~nm}$ of $\mathrm{N}, \mathrm{N}^{\prime}$ diphenyl-N, $\mathrm{N}^{\prime}$ bis(3-methylphenyl)-(1,1'-biphenyl)- 4,4'diamine (TPD) as the hole transport layer (HTL), $50 \mathrm{~nm}$ of $\mathrm{Alq}_{3}$ as an emissive/electron transport layer, and a $\operatorname{LiF}(1 \mathrm{~nm}) /$ $\mathrm{Al}(100 \mathrm{~nm})$ or $\mathrm{Al}(100 \mathrm{~nm})$ cathode. The TPD and $\mathrm{Alq}_{3}$ were purchased from Aldrich and purified using train sublimation prior to use. The final areas of the devices were $\sim 4 \mathrm{~mm}^{2}$. The ITO substrates were patterned using photolithography and cleaned by ultrasonicating in detergent/water, acetone, and chloroform. In order to vary the hole injection efficiency, 
we compared a device with a standard oxygen plasma treatment, device 1; with a device without a plasma treatment, device 2. Device 3 was used to change the electron injection efficiency and has a standard oxygen plasma treatment but an $\mathrm{Al}$ only cathode. The oxygen plasma treatment was performed in a Diener Electronic Femto Plasma-system at a plasma power of $30 \mathrm{~W}$ and with a 2.5 millibars oxygen pressure. The plasma treated substrate was immediately transferred to the deposition chamber for device fabrication. The deposition of the organic layers and metal electrodes were performed using a Kurt J. Lesker SPECTROS evaporation system with a base pressure during evaporation of $\sim 10^{-7}$ millibar. The rate of deposition of organic materials was about $0.2 \mathrm{~nm} / \mathrm{s}$, while that of the aluminium was varied from $\sim 0.1$ to $0.5 \mathrm{~nm} / \mathrm{s}$. A calibrated oscillating quartz crystal monitor was used to determine the deposition rate and thickness of the deposited layer. The whole device fabrication was performed without breaking vacuum.

Immediately after growth, the devices were placed in a light-tight sample holder with a calibrated silicon photodetector (Newport 818-SL), whose output is independent of magnetic field, placed on the top surface of the device. For the efficiency measurements, the device was place in to a sample holder inserted into an integrating sphere. Only emission from the front surface was measured and no attempt was made to correct for the total emission from the device. The sample holder was then placed between the poles of an electromagnet with the magnetic field perpendicular to the direction of current flow in the device. 32 independent measurements were taken and then averaged with the device operated in vacuum and in constant voltage mode. Before and after each field measurement, a measurement at null field was taken and used to remove any effects due to drift in the device characteristics. The measurements were performed using a Keithley 236 source-measure unit and Newport 1830 optical power meter. All measurements were performed at room temperature.

Figure 1 shows the efficiency against voltage for $50 \mathrm{~nm}$ $\mathrm{Alq}_{3}$ OLEDs at different plasma treatment conditions and cathodes. It can be observed that the peak efficiencies are $\sim 0.27 \%$ (device 1), $0.023 \%$ (device 2 ), and $\sim 0.015 \%$ (device 3 ), respectively. It is well known that treating ITO with an oxygen plasma has the effect of increasing device efficiency for OLEDs, and it has been suggested that this effect correlates with an increase in the work function resulting in increased hole injection. ${ }^{13-15}$ This is consistent with our observation where the device efficiency is reduced by an order of magnitude for device 2 compared to the optimised device 1. Given that the rest of the device fabrication remains the same, including the cathode, this is consistent with a reduction in the hole injection into the device. Device 3 (Al only device) also shows a decrease in efficiency, by approximately an order of magnitude, compared to device 1 and here the only difference is the removal of the LiF electron injection layer. The role of this $\mathrm{LiF}$ layer is to lower the effective work function of the cathode. ${ }^{16,17}$ Without the LiF layer, the electron injection is reduced and again the efficiency of OLEDs is decreased. We therefore have a range of devices where we have independently reduced either the hole or electron injection efficiency by approximately an order of magnitude.

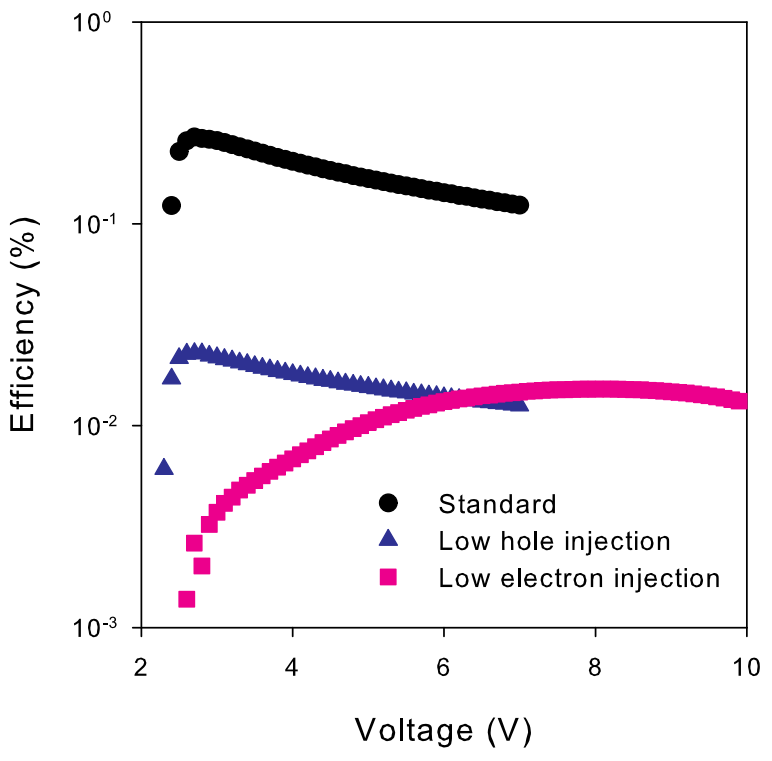

FIG. 1. Device efficiency versus voltage for the standard, low hole injection, and low electron injection devices.

Figure 2 shows the OMR curves (plotted as the relative change in current) for the three devices at an operating current of $\sim 30 \mu \mathrm{A}$. Gillin et al. ${ }^{18,19}$ reported that the OMR data for a $50 \mathrm{~nm} \mathrm{Alq}_{3}$ device can be fitted using the TPI model that includes two independent processes, namely, the exciton trapping and the triplet polaron interaction components. This TPI model can be represented by a double Lorentzian equation as follows:

$$
f(B)=a_{t} \frac{B^{2}}{\left(B^{2}+B_{t}^{2}\right)}+a_{i} \frac{B^{2}}{\left(B^{2}+B_{i}^{2}\right)},
$$

where $B$ is the applied magnetic field, $a_{t}$ an $a_{i}$ are the prefactors for the Lorentzians, and $B_{t}$ and $B_{i}$ are the saturation fields; the subscripts $t$ and $i$ stand for trapping and interaction, respectively. The constraints used in the fit, taken from Ref. 18 , were $5 \mathrm{mT}<B_{t}<7 \mathrm{mT}, a_{t}>0, B_{i}=160 \mathrm{mT}$, and $a_{i}>0$.

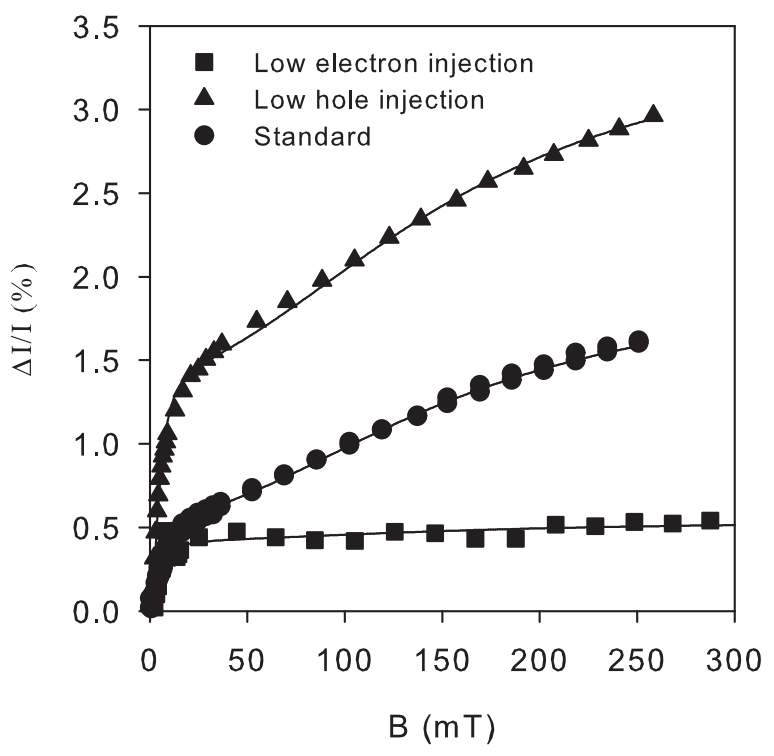

FIG. 2. The percentage change in current, at a drive current of $\sim 30 \mu \mathrm{A}$, for the standard, low hole injection, and low electron injection devices. 
TABLE I. The ratio of the site blocking (weak trapping) term, $a_{t}$, to the triplet polaron interaction term, $a_{i}$, for the three devices at different operating currents. Note that the device area is equal for all devices.

\begin{tabular}{llllll}
\hline \hline & \multicolumn{5}{c}{$a_{t} / a_{i}$} \\
\cline { 2 - 6 } & $\mathrm{I} \sim 1 \mu \mathrm{A}$ & $\mathrm{I} \sim 5 \mu \mathrm{A}$ & $\mathrm{I} \sim 30 \mu \mathrm{A}$ & $\mathrm{I} \sim 100 \mu \mathrm{A}$ & $\mathrm{I} \sim 300 \mu \mathrm{A}$ \\
\hline Device 1 & 1.36 & 0.58 & 0.41 & 0.35 & 0.35 \\
Device 2 & 1.25 & 1.03 & 0.72 & 0.56 & 0.47 \\
Device 3 & 5.2 & 2.3 & 3.8 & 3.2 & 2.3 \\
\hline \hline
\end{tabular}

From Figure 2, it can be seen that using this double Lorentzian gives an excellent fit to the data for all devices, despite the differences in electron and hole balance between them. However, although the qualities of the fits are good in each case, there are important differences that can be seen. For the standard device (device 1) and the reduced hole device (device 2), the OMR curves appear to be very similar in shape and both show a distinct low field and high field component, which we have previously attributed to site blocking (or weak trapping) and triplet polaron interactions. For the electron deficient device, however, the OMR curves are dominated by the site blocking (weak trapping) component and TPI plays a much smaller role. Indeed, if one compares the ratio of the magnitude of the two components, given by the two prefactors in Eq. (1), then it can be seen that the ratio $a_{t} / a_{i}$ increases by up to a factor of 4 between the devices with good electron injection (device 1 and device 2) and the device with poor electron injection (device 3) (Table I). This ratio is independent of whether one compares devices at equal operating currents, as shown in Figure 2, or voltages. Figure 3 shows the two prefactors from Eq. (1) (plotted as absolute change in current) for each device as a function of operating current. It can be seen that the magnitude of the trapping terms, $a_{t}$, is comparable for all devices regardless of current density. For the TPI terms, $a_{i}$, the values are comparable for both the standard and low hole injection device, whereas for the low electron injection device the interaction component is significantly reduced in magnitude. This dramatic reduction in the TPI contribution to OMR for the low electron injection device indicates that the TPI component is being dominated by the electron-current in the $\mathrm{Alq}_{3}$ system. According to the TPI model, this component is related to polarons that are strongly bound to triplets, such as through the production of trions. If the mobility of electrons and holes are equal, then the probability of any given electron or hole finding a triplet is equal. However, in $\mathrm{Alq}_{3}$, the hole mobility is approximately two orders of magnitude smaller than that for electrons. ${ }^{20-24}$ Therefore, the interaction frequency of triplets and polarons would be expected to be higher for electrons than holes, assuming balanced charge concentrations (i.e., they travel further in a given time so are more likely to encounter a triplet site). Therefore, reducing the electron concentration might be expected to reduce the magnitude of the TPI. However, given that the magnitude of the trapping term, $a_{t}$, is independent of the electron of hole injection, this demonstrates that the probability for both electrons and holes to meet with a triplet is identical. Therefore, the reduced magnitude for the TPI interaction term as the electron injection is reduced is indicative that the TPI interaction

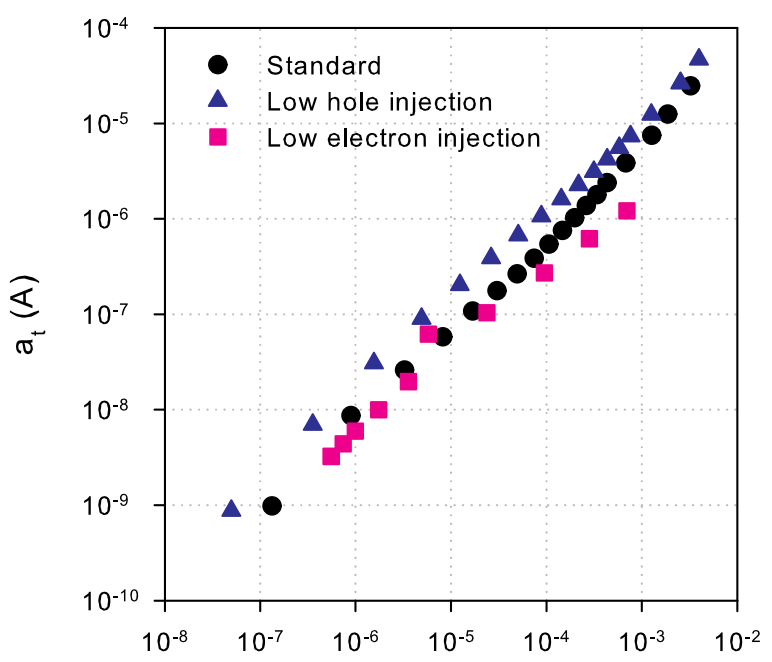

I (A)

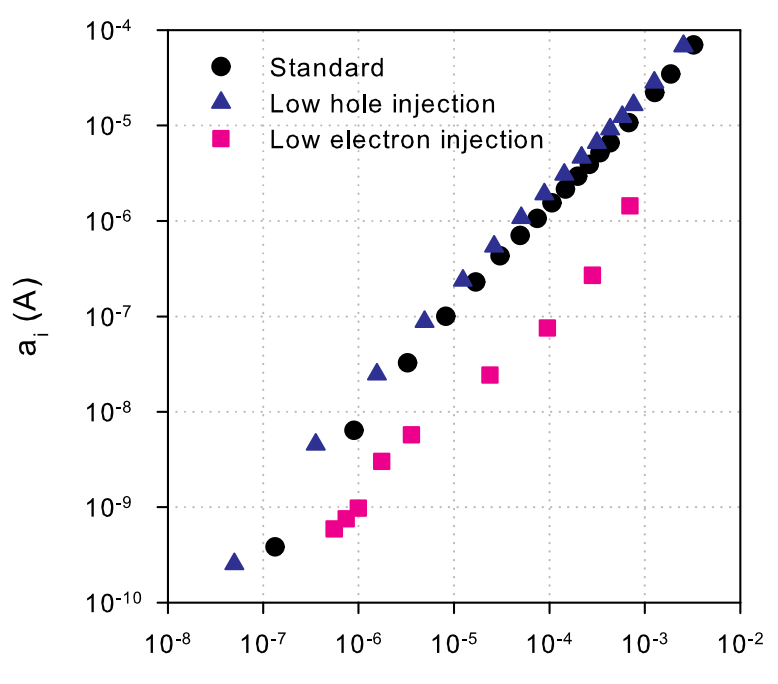

I (A)

FIG. 3. The magnitude of the prefactors in Eq. (1) (plotted as change in absolute current) as a function of the device current for the standard, low hole injection, and low electron injection devices.

for electrons is intrinsically larger than that for holes. This may be, for example, because the triplet represents a deeper trap for electrons compared to holes.

In conclusion, we have investigated the effect of changing the electron/hole balance on the OMR of an $\mathrm{Alq}_{3}$ OLED and found that whilst reducing the hole current in the device has relatively little effect of the OMR, despite an order of magnitude reduction in the device efficiency; when the electron current is reduced by a similar amount, the OMR changes dramatically with the TPI component being reduced by approximately an order of magnitude. These results suggest that the TPI contribution in $\mathrm{Alq}_{3}$ is dominated by the formation of electron-trions as opposed to hole-trions.

We are grateful for financial support from National Natural Science Foundation of China (Grant No. 61307039).

${ }^{1}$ J. Kalinowski, M. Cocchi, D. Virgili, P. Di Marco, and V. Fattori, Chem. Phys. Lett. 380, 710 (2003).

${ }^{2}$ O. Mermer, G. Veeraraghavan, T. L. Francis, and M. Wohlgenannt, Solid State Commun. 134(9), 631-636 (2005). 
${ }^{3}$ V. N. Prigodin, J. D. Bergeson, D. M. Lincoln, and A. J. Epstein, Synth. Met. 156, 757 (2006).

${ }^{4}$ Y. Wu and B. Hu, Appl. Phys. Lett. 89, 203510 (2006).

${ }^{5}$ H. Odaka, Y. Okimoto, T. Yamada, H. Okamoto, M. Kawasaki, and Y. Tokura, Appl. Phys. Lett. 88, 123501 (2006).

${ }^{6}$ P. Desai, P. Shakya, T. Kreouzis, and W. P. Gillin, Phys. Rev. B 76, 235202 (2007).

${ }^{7}$ F. L. Bloom, W. Wagemans, M. Kemerink, and B. Koopmans, Phys. Rev. Lett. 99, 257201 (2007).

${ }^{8}$ B. F. Ding, Y. Q. Zhan, Z. Y. Sun, X. M. Ding, X. Y. Hou, Y. Z. Wu, I. Bergenti, and V. Dediu, Appl. Phys. Lett. 93, 183307 (2008).

${ }^{9}$ L. Y. Xin, C. N. Li, F. Li, S. Y. Liu, and B. Hu, Appl. Phys. Lett. 95, 123306 (2009).

${ }^{10}$ S. Zhang, N. J. Rolfe, P. Desai, P. Shakya, A. J. Drew, T. Kreouzis, and W. P. Gillin, Phys. Rev. B 86(7), 075206 (2012).

${ }^{11}$ P. A. Bobbert, T. D. Nguyen, F. W. A van Oost, B. Koopmans, and M. Wohlgenannt, Phys. Rev. Lett. 99, 216801 (2007).

${ }^{12}$ R. N. Mahato, H. Lülf, M. H. Siekman, S. P. Kersten, P. A. Bobbert, M. P. de Jong, L. De Cola, and W. G. van der Wiel, Science 341(6143), 257 (2013).

${ }^{13}$ D. J. Milliron, I. G. Hill, C. Shen, A. Kahn, and J. Schwartz, J. Appl. Phys. 87(1), 572-576 (2000).
${ }^{14}$ J. S. Kim, M. Granstrom, R. H. Friend, N. Johansson, W. R. Salaneck, R. Daik, W. J. Feast, and F. Cacialli, J. Appl. Phys. 84(12), 6859-6870 (1998).

${ }^{15}$ I. G. Hill, D. Milliron, J. Schwartz, and A. Kahn, Appl. Surf. Sci. 166(1-4), 354-362 (2000).

${ }^{16}$ T. M. Brown, R. H. Friend, I. S. Millard, D. J. Lacey, J. H. Burroughes, and F. Cacialli, Appl. Phys. Lett. 79(2), 174-176 (2001).

${ }^{17}$ T. M. Brown, I. S. Millard, D. J. Lacey, J. H. Burroughes, R. H. Friend, and F. Cacialli, Synth. Met. 124(1), 15-17 (2001).

${ }^{18}$ W. P. Gillin, S. J. Zhang, N. J. Rolfe, P. Desai, P. Shakya, A. J. Drew, and T. Kreouzis, Phys. Rev. B 82, 195208 (2010).

${ }^{19}$ S. J. Zhang, A. J. Drew, T. Kreouzisa, and W. P. Gillin, Synth. Met. 161, 628 (2011).

${ }^{20}$ R. G. Kepler, P. M. Beeson, S. J. Jacobs, R. A. Anderson, M. B. Sinclair, V. S. Valencia, and P. A. Cahill, Appl. Phys. Lett. 66(26), 3618 (1995).

${ }^{21}$ T. Tsutsui, H. Tokuhisa, and M. Era, Polym. Photon. Devices 3281, 230 (1998).

${ }^{22}$ S. Naka, H. Okada, H. Onnagawa, Y. Yamaguchi, and T. Tsutsui, Synth. Met. 111-112, 331 (2000).

${ }^{23}$ H. H. Fong and S. K. So, J. Appl. Phys. 100(9), 094502 (2006).

${ }^{24}$ R. L. Martin, J. D. Kress, I. H. Campbell, and D. L. Smith, Phys. Rev. B 61(23), 15804 (2000). 